\title{
Clinicopathological significance of lipocalin 2 nuclear expression in invasive breast
}

cancer

Sasagu Kurozumi ${ }^{1,2}$, Sami Alsaeed ${ }^{1}$, Nnamdi Orah $^{1}$, Islam M. Miligy ${ }^{1}$, Chitra Joseph ${ }^{1}$, Abrar Aljohani ${ }^{1}$, Michael S. Toss ${ }^{1}$, Takaaki Fujii², Ken Shirabe², Andrew R. Green ${ }^{1}$, Mohammed A. Aleskandarany ${ }^{1}$ and Emad A. Rakha ${ }^{1}$

${ }^{1}$ Nottingham Breast Cancer Research Centre, Division of Cancer and Stem Cells, School of Medicine, University of Nottingham, Nottingham, United Kingdom

2Department of General Surgical Science, Gunma University Graduate School of Medicine, Gunma, Japan

\section{Corresponding author:}

Professor Emad Rakha

Department of Histopathology, Division of Cancer and Stem Cells, School of Medicine, The University of Nottingham and Nottingham University Hospitals NHS Trust, Nottingham City Hospital, Nottingham NG5 1PB, UK

Email: Emad.Rakha@nottingham.ac.uk 
PURPOSE: The epithelial-mesenchymal transition (EMT) plays a key role in breast cancer progression and metastasis. Lipocalin 2 (LCN2) is involved in the regulation of EMT. The aim of this study was to investigate the clinicopathological significance of LCN2 expression in breast cancer.

METHODS: The expression of LCN2 protein was immunohistochemically assessed in two well-characterised annotated cohorts of breast cancer (discovery cohort, $n=612$; validation cohort, $n=1,363$ ). The relationship of LCN2 expression and subcellular location with the clinicopathological factors and outcomes of patients was analysed.

RESULTS: Absent or reduced nuclear LCN2 expression was associated with features of aggressive behaviour, including high histological grade, high Nottingham Prognostic Index, high Ki67 labelling index, hormone receptor negativity and human epidermal growth factor receptor 2 positivity. The high cytoplasmic expression of LCN2 was correlated with lymph node positivity. The nuclear downregulation of LCN2 was correlated with the overexpression of EMT associated proteins (N-cadherin and Twist-related protein 2) and basal biomarkers (cytokeratin 5/6 and epidermal growth factor receptor). Unlike the cytoplasmic expression of LCN2, the loss of nuclear expression was a significant predictor of poor outcome. The combinatorial expression tumours with high cytoplasmic and low nuclear expression were associated with the worst prognosis.

CONCLUSIONS: Tumour cell expression of LCN2 plays a role in breast cancer progression with loss of its nuclear expression is associated with aggressive features and poor outcome. Further functional analysis is warranted to confirm the relationship between the subcellular localisation LCN2 and behaviour of breast cancer.

Keywords: Invasive breast cancer; lipocalin 2; epithelial-mesenchymal transition; N-cadherin; basal type

\section{INTRODUCTION}

Breast cancer is a heterogeneous disease with variable morphology, metastatic behaviour and response to therapy [1]. Further investigations of new biomarkers are warranted to develop a personalised management of 
this disease. Lipocalin 2 (LCN2) is a secreted glycoprotein that transports small lipophilic ligands and acts as an iron binding protein. It also acts as a member of the autocrine system via SLC22A17 (receptor of LCN2) in cancer cells [2-4]. LCN2 is expressed in the extracellular matrix of tumours, regulates the epithelialmesenchymal transition (EMT), and is involved in the proliferation of cancer cells $[5,6]$. Previous studies suggested that LCN2 expressed in stromal tissue may regulate tumour angiogenesis, oncogenesis and progression in various types of cancer by controlling EMT and proliferation [7, 8]. LCN2 has been associated with matrix metallopeptidase 9 (MMP9), which is an established factor related to EMT and tumour metastasis in the extracellular matrix [9]. When LCN2 forms a complex with MMP9, it increases MMP9-activity and prevents its auto-degradation. Since MMP9 degrades the extracellular matrix and basement membranes, LCN2/ MMP9 complex contributes to tumour progression, invasion and metastasis [9].

Abnormal expression of LCN2 plays important role in various pathological conditions including inflammation and tissue injury. LCN2 is also considered as a potential biomarker and a modulator of human epithelial malignancy. The expression of LCN2 is dysregulated in a variety of cancers including hepatocellular [8], pancreatic [10], colorectal [11], and prostate [12] carcinomas. In the breast, it has been reported that stromasecreted LCN2 promotes metastasis in vitro and in vivo and contributes to tumour progression [13]. Decreasing LCN2 expression has reduced the invasion and migration ability of HER2-positive breast cancer cells [14]. However, some authors have reported that overexpression of LCN2 does not affect cell proliferation or anchorage-independent growth in vitro, or primary tumor weight in vivo [15]. Data from The Cancer Genome Atlas (TCGA) using 1,075 cases indicates an association between loss of $L C N 2$ mRNA expression and poor prognosis [16]. Therefore, clinicopathological significance of LCN2 expression and its morphological characteristics a in breast cancer remain unclear. In the present study, we attempt to demonstrate the clinicopathological significance of LCN2 expression using immunohistochemistry (IHC). We assessed the relationship between LCN2 expression and its subcellular localisation and other clinicopathological factors, including breast cancer progression/metastasis-related biomarkers and breast cancer patient outcomes.

\section{MATERIALS AND METHODS}

\section{Patients` characteristics}

Two independent cohorts of patients with early invasive breast cancer were included in this study: a discovery set and a validation set comprising 612 and 1,363 patients, respectively. The clinicopathological significance of the nuclear, cytoplasmic and combined expression of LCN2 was evaluated using the former set. The clinicopathological and prognostic value of the nuclear expression of LCN2 was further validated using the 
latter set. All patients underwent primary breast surgery without prior neoadjuvant therapy at the Nottingham University Hospitals (Nottingham, UK). Clinical and pathological data of patients (i.e. age at diagnosis, histological tumour type, grade, tumour size, lymph node status, Nottingham Prognostic Index and lymphovascular invasion) were collected. Survival data, including breast cancer-specific survival (BCSS; defined as the time [in months] from the date of the primary surgical treatment to the time of death from breast cancer), were retrieved. Data related to the expression of basic breast cancer markers, including oestrogen receptor (ER), progesterone receptor (PR) and human epidermal growth factor 2 (HER2), are available [17-19].

The expression of a large panel of breast cancer progression/metastasis-related biomarkers, including the Ki67 labelling index, E-cadherin, N-cadherin, P-cadherin, Twist-related protein 2 (TWIST2) and basal markers (cytokeratin 5/6 [CK5/6] and epidermal growth factor receptor [EGFR]), was also studied in a previous IHC analysis using the discovery cohort [20-25].

\section{Immunohistochemistry}

The specificity of the LCN2 antibody (LS-C405956; Lifespan Biosciences, Seattle, WA) (dilution: 1:500) was validated, and a specific band $(70 \mathrm{kDa})$ was detected through western blotting of lysate obtained from the cell line HeLa (The American Type Culture Collection, Rockville, MD) (Supplementary Fig 1). Full-face tissue sections from patients with invasive breast cancer $(n=10)[24-26]$ were stained before tissue microarray (TMA) to assess the morphological characteristics of LCN2 expression and test the suitability of this method. TMA sections from the discovery and validation sets were IHC-stained to evaluate the expression of LCN2 protein. The H-score $[27,28]$ was used to assess the nuclear and cytoplasmic expression of LCN2.

\section{Statistical analysis}

SPSS statistical software version 24.0 (IBM Corp., Armonk, NY) was used for statistical analysis. The $\chi^{2}$ and Fisher's exact tests were used to evaluate the relationships between LCN2 expression and clinicopathological factors. BCSS was used to assess the prognostic utility of LCN2 expression. The association with survival was evaluated using the Kaplan-Meier method and the log-rank test. Multivariate survival analyse was assessed using the Cox proportional hazards regression model. The median of the H-scores was used as a cutoff point to divide the samples into high- and low-LCN2 expression groups. A $p$-value $<0.05$ (two-tailed) denoted statistical significance.

\section{RESULTS}

Morphological characteristics of LCN2 protein expression 
The morphological features of LCN2 expression using full-face tissue sections of invasive breast cancer are shown in Figure 1. LCN2 showed staining signals in both the cytoplasm and nucleus of malignant epithelial cells, with weak staining noted in the stroma. The expression of LCN2 in the nuclei of adjacent apparently normal terminal duct-lobular units was moderate to strong. In contrast, the LCN2 nuclear immunoreactivity of invasive cancer cells was lower than that of adjacent normal epithelial cells. The immunoreactivity of LCN2 in the cytoplasm of cancer cells was moderate compared with that reported in the nucleus.

In this study, $310(51 \%)$ cases showed high cytoplasmic expression whereas $316(52 \%)$ cases showed high nuclear expression. Regarding subcellular localisation, cases were classified into four combinatorial phenotypic groups as follows: (1) low nuclear and high cytoplasmic expression (21\%); (2) high nuclear and low cytoplasmic expression (19\%); (3) both with low expression (30\%); and (4) both with high expression (29\%) (Fig 2).

\section{Clinicopathological characteristics of LCN2 protein expression}

The low nuclear expression of LCN2 was significantly associated with high histological grade $(p<0.0001)$, high Nottingham Prognostic Index $(p=0.00055)$, ER negativity $(p<0.0001)$, PR negativity $(p=0.0013)$ and breast cancer subtypes ( $p=0.00034$ ) (Table 1 ). A total of $60 \%$ of HER2-positive and 65\% of triple-negative cases showed a low nuclear expression of LCN2. Low nuclear expression was significantly associated with high Ki67 labelling index $(p<0.0001)$, high N-cadherin expression $(p=0.00052)$, high TWIST2 expression $(p=$ $0.039), \mathrm{CK} 5 / 6$ positivity $(p=0.0088)$ and EGFR positivity $(p=0.019)$. However, no correlation was identified between the nuclear expression of LCN2 and E-cadherin (Table 2). In addition, the low nuclear expression of LCN2 was associated with a shorter BCSS $\left(X^{2}=4.90, p=0.027\right)$ (Fig 3a).

The high cytoplasmic expression of LCN2 was significantly correlated with lymph node metastasis $(p=0.039$; Table 1) and expression of E-cadherin ( $p=0.010$; Table 2). However, the cytoplasmic expression of LCN2 was not a significant predictor of outcome (Supplementary Fig 2).

\section{Validation analysis for the clinicopathological significance of LCN2 nuclear expression}

In the validation cohort, the low nuclear expression of LCN2 was significantly associated with high histological grade $(p<0.0001)$, high Nottingham Prognostic Index $(p<0.0001)$, ER negativity $(p<0.0001)$ and PR negativity $(p<0.0001)$. The downregulation of the nuclear expression of LCN2 was also significantly related to 
large tumour size $(p=0.00066)$ and HER2 positivity ( $p=0.0042$; Supplementary Table 1$)$. A total of $75 \%$ of triple-negative cases showed a low nuclear expression of LCN2 $(p<0.0001)$ (Supplementary Table 1).

The significance of the low nuclear expression of LCN2 as a poor prognostic marker was validated in 1,363 cases $\left(\chi^{2}=11.66, p=0.00064\right)$ (Fig 3b), although the low nuclear expression of LCN2 was not an independent prognostic marker in the multivariate analysis (Supplementary Table 2)

\section{Combinatorial expression}

Although no significant difference in the survival between these four groups, the tumours with low cytoplasmic and high nuclear expression showed the best outcome whereas the tumours with high cytoplasmic and low nuclear expression showed the worst outcome (Supplementary Fig 3).

\section{DISCUSSION}

EMT plays a key role in the metastasis of breast cancer [29]. Several previous studies have indicated the possible value of biomarkers associated with EMT as prognostic factors and have proposed their utility in predicting the response of invasive breast cancer to chemotherapy [30, 31]. In the present study, a low nuclear expression of LCN2 was significantly associated with a high expression of N-cadherin and TWIST2. Ncadherin, a member of the genetically related transmembrane glycoproteins, promotes tumour-stroma interactions and stimulates cell motility, invasion and metastasis [32, 33]. The overexpression of TWIST2 promotes metastasis of breast cancer by activating EMT and enhancing the self-renewal system of cancer stemlike cells [34]. Previous studies have suggested that LCN2 regulates the activity of the Ras pathway and regulates cell migration and EMT $[35,36]$. Further functional studies are necessary to explore the association of aberrant LCN2 function with EMT in invasive breast cancer.

In the present study, the low nuclear expression of LCN2 was significantly associated with the positive expression of basal markers such as CK5/6 and EGFR. Several studies have suggested a strong correlation between the expression of basal cytokeratins and cell proliferation [37, 38]. EGFR is not a basal-like specific marker like CK5/6; however, it is expressed in the basal-like type and strongly assists in the immunohistochemical identification of this type [39, 40]. The Nottingham Prognostic Index + is based on the assessment of the biological class combined with established clinicopathological prognostic variables, including CK5/6 and EGFR. This index provides improved stratification of patient outcomes for invasive breast cancer [41]. Recently, the use of molecular-targeted therapy against EGFR-positive breast cancer (e.g. neratinib [42], 
pertuzumab [43] and lapatinib [44]) has been proposed. The association of LCN2 expression with basal-like breast cancers may define a subset of patients more likely to benefit from treatment with these agents.

Several previous studies investigating the complex role of LCN2 in a variety of cancer types have suggested that LCN2 leads to apoptosis and suppresses proliferation/metastasis $[8,10,45,46]$; however, a number of other studies have suggested that the expression of LCN2 promotes tumour growth, migration and invasion [47, 48]. The low nuclear expression of LCN2 has been significantly related to high tumour proliferation, hormonal receptor negativity, HER2 positivity and poor prognostic outcome. Notably, other studies have indicated that the high cytoplasmic expression of LCN2 is associated with the decreased disease-free survival in patients with invasive breast cancer [49, 50]. In the present study, the cytoplasmic expression of LCN2 was related to positivity for lymph node metastasis. The tumour microenvironment controls the LCN2-autocrine system of cancer cells via endoplasmic reticulum stress-dependent and -independent mechanisms [51]. Once released in the extracellular compartment, LCN2 drives iron sequestration and internalisation through established receptors, promoting cell survival and EMT $[48,51]$. These mechanisms may be responsible for the clinicopathologically significant discrepancy observed between the nuclear and cytoplasmic expression of LCN2. Therefore, further investigations are warranted to examine the activity and functions of LCN2 based on its intracellular localisation.

\section{CONCLUSIONS}

Not only the expression but also subcellular localisation of LCN2 expression appears to play a role in breast cancer progression. Loss or reduced expression of nuclear LCN2 expression is related to the aggressive types and poor outcome in breast cancer. Further functional studies of LCN2 in breast cancer with consideration of its subcellular localisation in tumour cells are warranted.

\section{Acknowledgements}

The authors thank the Breast Cancer Now Tissue Bank and the University of Nottingham and Pathological Society of Great Britain and Ireland. 


\section{Compliance with ethical standards}

\section{Funding}

This research was funded by the Pathological Society Trainees Small Grant Application (No: 2201) and the University of Nottingham (Nottingham Life Cycle 6).

\section{Disclosure of potential conflicts of interest}

KS has received research grants from CHUGAI Pharmaceutical Co., Ltd. and Ono Pharmaceutical Co., Ltd. The remaining authors declare that they have no conflicts of interest.

\section{Research involving human participants}

This study was approved by the Nottingham Research Ethics Committee 2 (Reference title: Development of a molecular genetic classification of breast cancer). All procedures performed in studies involving human participants were in accordance with the ethical standards of the institutional and/or national research committee, and with the 1964 Helsinki declaration and its later amendments or comparable ethical standards. This article does not contain any studies with animals performed by any of the authors. 


\section{Informed consent}

Informed consent was obtained from all participants in the study.

\section{REFERENCES}

1. Cancer Research UK (2019) Breast cancer statistics/Statistics by cancer type/ Cancer Statistics for the UK. https://www.cancerresearchuk.org/health-professional/cancer-statistics/statistics-by-cancer-type/breastcancer. Accessed 04 June 2019

2. Cheng G, Sun X, Wang J et al (2014) HIC1 silencing in triple-negative breast cancer drives progression through misregulation of LCN2. Cancer Res 74:862-872

3. Wang HH, Wu MM, Chan MW et al (2014) Long-term low-dose exposure of human urothelial cells to sodium arsenite activates lipocalin-2 via promoter hypomethylation. Arch Toxicol 88:1549-1559

4. Ören B, Urosevic J, Mertens C et al (2016) Tumour stroma-derived lipocalin-2 promotes breast cancer metastasis. J Pathol 239:274-285

5. Yang J, McNeish B, Butterfield C et al (2013) Lipocalin 2 is a novel regulator of angiogenesis in human breast cancer. FASEB J 27:45-50

6. Liao CJ, Li PT, Lee YC et al (2013) Lipocalin 2 induces the epithelial-mesenchymal transition in stressed endometrial epithelial cells: possible correlation with endometriosis development in a mouse model. Reproduction 147:179-187

7. Kim SL, Lee ST, Min IS et al (2017) Lipocalin 2 negatively regulates cell proliferation and epithelial to mesenchymal transition through changing metabolic gene expression in colorectal cancer. Cancer Sci 108:2176-2186 
8. Wang YP, Yu GR, Lee MJ et al (2013) Lipocalin-2 negatively modulates the epithelial-to-mesenchymal transition in hepatocellular carcinoma through the epidermal growth factor (TGF-beta1)/Lcn2/Twist1 pathway. Hepatology 58:1349-1361

9. Fernández CA, Yan L, Louis G et al (2005) The matrix metalloproteinase-9/neutrophil gelatinase-associated lipocalin complex plays a role in breast tumor growth and is present in the urine of breast cancer patients. Clin Cancer Res 11:5390-5395

10. Leung L, Radulovich N, Zhu CQ et al (2012) Lipocalin2 promotes invasion, tumorigenicity and gemcitabine resistance in pancreatic ductal adenocarcinoma. PLoS One7(10):e46677

11. Feng M, Feng J, Chen W et al (2016) Lipocalin2 suppresses metastasis of colorectal cancer by attenuating NF-кB-dependent activation of snail and epithelial mesenchymal transition. Mol Cancer 15:77

12. Tung MC, Hsieh SC, Yang SF et al (2013) Knockdown of lipocalin-2 suppresses the growth and invasion of prostate cancer cells. Prostate 73:1281-1290

13. Ören B, Urosevic J, Mertens C et al (2016) Tumour stroma-derived lipocalin-2 promotes breast cancer metastasis. J Pathol 239:274-285

14. Leng X, Ding T, Lin H et al (2009) Inhibition of lipocalin 2 impairs breast tumorigenesis and metastasis. Cancer Res 69:8579-8584

15. Shi H, Gu Y, Yang J et al (2008) Lipocalin 2 promotes lung metastasis of murine breast cancer cells. J Exp Clin Cancer Res 27:83

16. Uhlen M, Zhang C, Lee S (2017) A pathology atlas of the human cancer transcriptome. Science 357:eaan 2507

17. Aleskandarany MA, Abduljabbar R, Ashankyty I et al (2016) Prognostic significance of androgen receptor expression in invasive breast cancer: transcriptomic and protein expression analysis. Breast Cancer Res Treat 159:215-227

18. Rakha EA, Agarwal D, Green AR et al (2017) Prognostic stratification of oestrogen receptor-positive HER2-negative lymph node-negative class of breast cancer. Histopathology 70:622-631

19. Rakha EA, Elsheikh SE, Aleskandarany MA et al (2009) Triple-negative breast cancer: distinguishing between basal and nonbasal subtypes. Clin Cancer Res 15:2302-2310

20. Green AR, Powe DG, Rakha EA et al (2013) Identification of key clinical phenotypes of breast cancer using a reduced panel of protein biomarkers. Br J Cancer 109:1886-1894

21. Rakha EA, Soria D, Green AR et al (2014) Nottingham Prognostic Index Plus (NPI+): a modern clinical decision making tool in breast cancer. Br J Cancer 110:1688-1697 
22. Aleskandarany MA, Green AR, Ashankyty I et al (2016) Impact of intratumoural heterogeneity on the assessment of Ki67 expression in breast cancer. Breast Cancer Res Treat 158:287-295

23. Muftah AA, Aleskandarany MA, Al-Kaabi MM et al (2017) Ki67 expression in invasive breast cancer: the use of tissue microarrays compared with whole tissue sections. Breast Cancer Res Treat 164:341-348

24. Kurozumi S, Joseph C, Sonbul S et al (2018) Clinicopathological and prognostic significance of Ras association and pleckstrin homology domains 1 (RAPH1) in breast cancer. Breast Cancer Res Treat 172(1):61-68

25. Kurozumi S, Joseph C, Sonbul S et al (2018) Clinical and biological roles of Kelch-like family member 7 in breast cancer: a marker of poor prognosis. Breast Cancer Res Treat 170:525-533

26. Kurozumi S, Joseph C, Raafat S et al (2019) Utility of Ankyrin 3 as a Prognostic Marker in AndrogenReceptor-Positive Breast Cancer. Breast Cancer Res Treat 176:63-73

27. McCarty KS Jr, Miller LS et al (1985) Estrogen receptor analyses. Correlation of biochemical and immunohistochemical methods using monoclonal antireceptor antibodies. Arch Pathol Lab Med 109:716-721

28. Detre S, Saclani Jotti G, Dowsett MA (1995) "Quickscore" method for immunohistochemical semiquantitation: validation for oestrogen receptor in breast carcinomas. J Clin Pathol 48:876-878

29. Wu Y, Sarkissyan M, Vadgama JV (2016) Epithelial-Mesenchymal Transition and Breast Cancer. J Clin Med 5:E13.

30. Lehmann BD, Bauer JA, Chen X et al (2011) Identification of human triple-negative breast cancer subtypes and preclinical models for selection of targeted therapies. J Clin Invest 121:2750-2767

31. Masuda H, Baggerly KA, Wang Y et al (2013) Differential response to neoadjuvant chemotherapy among 7 triple-negative breast cancer molecular subtypes. Clin Cancer Res 19:5533-5540

32. Nagi C, Guttman M, Jaffer S et al (2005) N-cadherin expression in breast cancer: correlation with an aggressive histologic variant--invasive micropapillary carcinoma. Breast Cancer Res Treat 94:225-235

33. Aleskandarany MA, Sonbul S, Surridge R et al (2017) Rho-GTPase activating-protein 18: a biomarker associated with good prognosis in invasive breast cancer. Br J Cancer 117:1176-1184

34. Fang X, Cai Y, Liu J et al (2011) Twist2 contributes to breast cancer progression by promoting an epithelialmesenchymal transition and cancer stem-like cell self-renewal. Oncogene 30:4707-4720

35. Hanai J, Mammoto T, Seth P et al (2005) Lipocalin 2 diminishes invasiveness and metastasis of Rastransformed cells. J Biol Chem 280:13641-13647

36. Yang J, Bielenberg DR, Rodig SJ et al (2009) Lipocalin 2 promotes breast cancer progression. Proc Natl Acad Sci U S A 106:3913-3918 
37. Sørlie T, Perou CM, Tibshirani R et al (2001) Gene expression patterns of breast carcinomas distinguish tumor subclasses with clinical implications. Proc Natl Acad Sci U S A 98:10869-10874

38. Perou CM, Sørlie T, Eisen MB et al (2000) Molecular portraits of human breast tumours. Nature 406:747-752

39. Nielsen TO, Hsu FD, Jensen K et al (2004) Immunohistochemical and clinical characterization of the basallike subtype of invasive breast carcinoma. Clin Cancer Res 10:5367-5374

40. Kurozumi S, Yamaguchi Y, Matsumoto H et al (2018) Comparing protein and mRNA expressions of the human epidermal growth factor receptor family in estrogen receptor-positive breast cancer. Med Mol Morphol 52:90-98.

41. Green AR, Soria D, Stephen J et al (2016) Nottingham Prognostic Index Plus: Validation of a clinical decision making tool in breast cancer in an independent series. J Pathol Clin Res 2:32-40

42. Martin M, Holmes FA, Ejlertsen B et al (2017) ExteNET Study Group (2017) Neratinib after trastuzumabbased adjuvant therapy in HER2-positive breast cancer (ExteNET): 5-year analysis of a randomised, double-blind, placebo-controlled, phase 3 trial. Lancet Oncol 18:1688-1700

43. Baselga J, Cortés J, Kim SB et al (2012) CLEOPATRA Study Group (2012) Pertuzumab plus trastuzumab plus docetaxel for metastatic breast cancer. N Engl J Med 366:109-119

44. Geyer CE, Forster J, Lindquist D et al (2006) Lapatinib plus capecitabine for HER2-positive advanced breast cancer. N Engl J Med 355:2733-2743

45. Lee EK, Kim HJ, Lee KJ et al (2011) Inhibition of the proliferation and invasion of hepatocellular carcinoma cells by lipocalin 2 through blockade of JNK and PI3K/Akt signaling. Int J Oncol 38:325-333

46. Leng X, Lin H, Ding T et al (2008) Lipocalin 2 is required for BCR-ABL-induced tumorigenesis. Oncogene 27:6110-6119

47. Feng M, Feng J, Chen W et al (2016) Lipocalin2 suppresses metastasis of colorectal cancer by attenuating

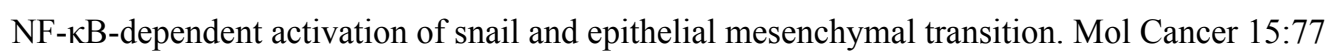

48. Leng X, Ding T, Lin H et al (2009) Inhibition of lipocalin 2 impairs breast tumorigenesis and metastasis. Cancer Res 69:8579-8584

49. Bauer M, Eickhoff JC, Gould MN et al (2008) (NGAL) is a predictor of poor prognosis in human primary breast cancer. Breast Cancer Res Treat 108:389-397

50. Wenners AS, Mehta K, Loibl S et al (2012) Neutrophil gelatinase-associated lipocalin (NGAL) predicts response to neoadjuvant chemotherapy and clinical outcome in primary human breast cancer. PLoS One $7: \mathrm{e} 45826$ 
51. Rodvold JJ, Mahadevan NR, Zanetti M (2012) Lipocalin 2 in cancer: when good immunity goes bad. Cancer Lett 316:132-138

\section{FIGURE LEGENDS}

Fig. 1 Immunohistochemical analysis of the morphological characteristics of LCN2

(a) The normal terminal duct-lobular unit showed a high immunoreactivity of LCN2 in the nuclei (magnification: $\times 200$ ). (b) The expression of LCN2 in the stromal cells was weak (magnification: $\times 100$ ). (c) The nuclear immunoactivity of LCN2 in invasive breast cancer cells was weaker than that observed in normal epithelial cells. However, the cytoplasmic immunoreactivity of LCN2 in invasive breast cancer cells was moderate (magnification: $\times 200)$.

Fig. 2 Representative tissue microarray images of the expression of LCN2

(a) Low nuclear and low cytoplasmic expression of LCN2; (b) low nuclear and high cytoplasmic expression of LCN2; (c) high nuclear and low cytoplasmic expression of LCN2; and (d) high nuclear and high cytoplasmic expression of LCN2. Magnification: $\times 200$ for all images.

Fig. 3 Breast cancer-specific survival stratified according to the nuclear expression of LCN2

(a) In the discovery cohort, breast cancer-specific survival was significantly worse in the LCN2-low expression group versus the LCN2-high expression group. (b) For the validation cohort, a significant difference was observed in the breast cancer-specific survival between LCN2-high and -low expression tumours. 
Table 1 Associations between LCN2 nuclear/cytoplasmic expression and clinicopathological features

\begin{tabular}{|c|c|c|c|c|c|c|}
\hline \multirow{3}{*}{ Factors } & \multicolumn{3}{|c|}{ Nuclear expression } & \multicolumn{3}{|c|}{ Cytoplasmic expression } \\
\hline & High LCN2 & Low LCN2 & \multirow{2}{*}{$p$-value } & High LCN2 & Low LCN2 & \multirow{2}{*}{$p$-value } \\
\hline & $\mathbf{N}(\%)$ & $\mathbf{N}(\%)$ & & $\mathbf{N}(\%)$ & $\mathbf{N}(\%)$ & \\
\hline \multicolumn{7}{|c|}{ Tumour size } \\
\hline$<2.0 \mathrm{~cm}$ & $150(52.3 \%)$ & $137(47.7 \%)$ & \multirow{2}{*}{0.075} & $147(51.2 \%)$ & $140(48.8 \%)$ & \multirow{2}{*}{0.81} \\
\hline$\geq 2.0 \mathrm{~cm}$ & $146(44.9 \%)$ & $179(55.1 \%)$ & & $163(50.2 \%)$ & $140(48.8 \%)$ & \\
\hline \multicolumn{7}{|c|}{ Nodal status } \\
\hline Negative & $181(48.8 \%)$ & $190(51.2 \%)$ & \multirow{2}{*}{0.80} & $175(47.2 \%)$ & $196(52.8 \%)$ & \multirow{2}{*}{0.039} \\
\hline Positive & $115(47.7 \%)$ & $126(52.3 \%)$ & & $135(56.0 \%)$ & $106(44.0 \%)$ & \\
\hline \multicolumn{7}{|c|}{ Nottingham Prognostic Index } \\
\hline Good prognostic group & $109(60.2 \%)$ & $72(39.8 \%)$ & \multirow{3}{*}{0.00055} & $89(49.2 \%)$ & $92(50.8 \%)$ & \multirow{3}{*}{0.21} \\
\hline Moderate prognostic group & $149(44.3 \%)$ & $187(55.7 \%)$ & & $165(49.1 \%)$ & $171(50.9 \%)$ & \\
\hline Poor prognostic group & $38(40.0 \%)$ & $57(60.0 \%)$ & & $56(58.9 \%)$ & $39(41.1 \%)$ & \\
\hline \multicolumn{7}{|c|}{ Histological grade } \\
\hline Grades 1 and 2 & $189(58.7 \%)$ & $133(41.3 \%)$ & \multirow{2}{*}{$<0.0001$} & $156(48.4 \%)$ & $166(51.6 \%)$ & \multirow{2}{*}{0.26} \\
\hline Grade 3 & $107(36.9 \%)$ & $183(63.1 \%)$ & & $154(53.1 \%)$ & $136(46.9 \%)$ & \\
\hline \multicolumn{7}{|c|}{ Lymphovascular invasion } \\
\hline Negative & $174(50.9 \%)$ & $168(49.1 \%)$ & \multirow{2}{*}{0.17} & $170(49.7 \%)$ & $172(50.3 \%)$ & \multirow{2}{*}{0.63} \\
\hline Positive & $122(45.2 \%)$ & $148(54.8 \%)$ & & $140(51.9 \%)$ & $130(48.1 \%)$ & \\
\hline \multicolumn{7}{|c|}{ Oestrogen receptor (ER) } \\
\hline Negative & $49(33.6 \%)$ & $97(66.4 \%)$ & -0 0001 & $74(50.7 \%)$ & $72(49.3 \%)$ & 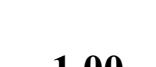 \\
\hline
\end{tabular}




\begin{tabular}{|c|c|c|c|c|c|c|}
\hline Positive & $247(53.0 \%)$ & $219(47.0 \%)$ & 00 & $236(50.6 \%)$ & $230(49.4 \%)$ & 10 \\
\hline \multicolumn{7}{|c|}{ Progesterone receptor (PR) } \\
\hline Negative & $99(40.2 \%)$ & $147(59.8 \%)$ & \multirow{2}{*}{0.0013} & $116(47.2 \%)$ & $130(52.8 \%)$ & \multirow{2}{*}{0.16} \\
\hline Positive & $197(53.8 \%)$ & $169(46.2 \%)$ & & $194(53.0 \%)$ & $172(47.0 \%)$ & \\
\hline \multicolumn{7}{|c|}{ Human epidermal growth factor receptor 2 (HER2) } \\
\hline Negative & $260(49.9 \%)$ & $261(50.1 \%)$ & \multirow{2}{*}{0.070} & $267(51.2 \%)$ & $254(48.8 \%)$ & \multirow{2}{*}{$\mathbf{0 . 5 0}$} \\
\hline Positive & $36(39.6 \%)$ & $55(60.4 \%)$ & & $43(47.3 \%)$ & $48(52.7 \%)$ & \\
\hline \multicolumn{7}{|c|}{ Breast cancer subtypes } \\
\hline ER-and/or PR-positive/HER2-negative & $223(53.9 \%)$ & $191(46.1 \%)$ & \multirow{3}{*}{0.00034} & $216(52.2 \%)$ & $198(47.8 \%)$ & \multirow{3}{*}{0.55} \\
\hline HER2-positive & $36(39.6 \%)$ & $55(60.4 \%)$ & & $43(47.3 \%)$ & $48(52.7 \%)$ & \\
\hline Triple negative & $37(34.6 \%)$ & $70(65.4 \%)$ & & $51(47.7 \%)$ & $56(52.3 \%)$ & \\
\hline
\end{tabular}


Table 2 Association of LCN2 nuclear/cytoplasmic expression with the expression of breast cancer progression/metastasis-related biomarkers

\begin{tabular}{|c|c|c|c|c|c|c|}
\hline \multirow{3}{*}{ Factors } & \multicolumn{3}{|c|}{ Nuclear expression } & \multicolumn{3}{|c|}{ Cytoplasmic expression } \\
\hline & High LCN2 & Low LCN2 & \multirow{2}{*}{$p$-value } & High LCN2 & Low LCN2 & \multirow{2}{*}{$p$-value } \\
\hline & $\mathbf{N}(\%)$ & $\mathbf{N}(\%)$ & & $\mathbf{N}(\%)$ & $\mathbf{N}(\%)$ & \\
\hline \multicolumn{7}{|c|}{ Ki67 labelling index } \\
\hline Low $(<10 \%)$ & $110(59.5 \%)$ & $75(40.5 \%)$ & \multirow{2}{*}{$<0.0001$} & $93(50.3 \%)$ & $92(49.7 \%)$ & \multirow{2}{*}{0.93} \\
\hline $\operatorname{High}(\geq 10 \%)$ & $114(40.1 \%)$ & $170(59.9 \%)$ & & $145(51.1 \%)$ & $139(48.9 \%)$ & \\
\hline \multicolumn{7}{|c|}{ E-cadherin } \\
\hline Low & $150(45.3 \%)$ & $181(54.7 \%)$ & \multirow{2}{*}{0.099} & $151(45.6 \%)$ & $180(54.4 \%)$ & \multirow{2}{*}{0.010} \\
\hline High & $135(52.3 \%)$ & $126(47.7 \%)$ & & $149(56.4 \%)$ & $115(43.6 \%)$ & \\
\hline \multicolumn{7}{|c|}{ N-cadherin } \\
\hline Low & $83(60.6 \%)$ & $54(39.4 \%)$ & \multirow{2}{*}{0.00052} & $75(54.7 \%)$ & $62(45.3 \%)$ & \multirow{2}{*}{0.48} \\
\hline High & $140(42.7 \%)$ & $188(57.3 \%)$ & & $167(50.9 \%)$ & $161(49.1 \%)$ & \\
\hline \multicolumn{7}{|c|}{ P-cadherin } \\
\hline Low & $117(43.7 \%)$ & $151(56.3 \%)$ & \multirow{2}{*}{0.66} & $137(51.1 \%)$ & $131(48.9 \%)$ & \multirow{2}{*}{0.79} \\
\hline High & $109(45.8 \%)$ & $129(54.2 \%)$ & & $125(52.5 \%)$ & $113(47.5 \%)$ & \\
\hline \multicolumn{7}{|c|}{ TGF- $\beta$} \\
\hline Low & $92(44.4 \%)$ & $115(55.6 \%)$ & \multirow{2}{*}{1.00} & $100(48.3 \%)$ & $107(51.7 \%)$ & \multirow{2}{*}{0.21} \\
\hline High & $134(44.8 \%)$ & $165(55.2 \%)$ & & $162(54.2 \%)$ & $137(45.8 \%)$ & \\
\hline \multicolumn{7}{|c|}{ TWIST2 } \\
\hline Low & $106(58.6 \%)$ & $75(41.4 \%)$ & \multirow{2}{*}{0.039} & $97(53.6 \%)$ & $84(46.4 \%)$ & \multirow{2}{*}{0.92} \\
\hline High & $118(48.2 \%)$ & $127(51.8 \%)$ & & $129(52.7 \%)$ & $116(47.3 \%)$ & \\
\hline
\end{tabular}




\begin{tabular}{|c|c|c|c|c|c|c|}
\hline \multicolumn{7}{|c|}{ CK5/6 } \\
\hline Negative & $257(51.1 \%)$ & $246(48.9 \%)$ & \multirow{2}{*}{0.0088} & $256(50.9 \%)$ & $247(49.1 \%)$ & \multirow{2}{*}{1.00} \\
\hline Positive & $37(36.6 \%)$ & $64(63.4 \%)$ & & $51(50.5 \%)$ & $50(49.5 \%)$ & \\
\hline \multicolumn{7}{|c|}{ EGFR } \\
\hline Negative & $245(51.0 \%)$ & $235(49.0 \%)$ & \multirow{2}{*}{0.019} & $248(51.7 \%)$ & $232(48.3 \%)$ & \multirow{2}{*}{0.61} \\
\hline Positive & $47(38.8 \%)$ & $74(61.2 \%)$ & & $59(48.8 \%)$ & $62(51.2 \%)$ & \\
\hline
\end{tabular}


Supplementary Table 2 Survival analysis based on clinicopathological characteristics of breast cancer, including LCN2 nuclear expression

\begin{tabular}{|c|c|c|c|c|}
\hline \multirow{2}{*}{\multicolumn{2}{|c|}{ Factors }} & \multicolumn{3}{|c|}{ Multivariate analysis } \\
\hline & & Hazard Ratio & $95 \% \mathrm{CI}$ & $p$ value \\
\hline \multirow{2}{*}{ LCN2 nuclear expression } & Low & \multicolumn{3}{|c|}{ Reference } \\
\hline & High & 0.88 & $0.68-1.15$ & 0.36 \\
\hline \multirow{2}{*}{ Tumour size } & $<2 \mathrm{~cm}$ & \multicolumn{3}{|c|}{ Reference } \\
\hline & $\geq 2 \mathrm{~cm}$ & 1.38 & $1.05-1.79$ & 0.018 \\
\hline \multirow{2}{*}{ Nodal status } & Negative & \multicolumn{3}{|c|}{ Reference } \\
\hline & Positive & 2.11 & $1.79-2.48$ & $<0.0001$ \\
\hline \multirow{2}{*}{ Histological grade } & Low & \multicolumn{3}{|c|}{ Reference } \\
\hline & High & 1.91 & $1.51-2.41$ & $<0.0001$ \\
\hline
\end{tabular}


Supplementary Table 1 Associations between LCN2 nuclear expression and clinicopathological features in the validation set

\begin{tabular}{|c|c|c|c|}
\hline \multirow{2}{*}{ Factors } & High LCN2 (nuclear) & Low LCN2 (nuclear) & \multirow{2}{*}{$p$-value } \\
\hline & $\mathbf{N}(\%)$ & $\mathbf{N}(\%)$ & \\
\hline \multicolumn{4}{|c|}{ Tumour size } \\
\hline$<2.0 \mathrm{~cm}$ & $404(49.9 \%)$ & $406(50.1 \%)$ & \multirow{2}{*}{0.00066} \\
\hline$\geq 2.0 \mathrm{~cm}$ & $217(40.3 \%)$ & $321(59.7 \%)$ & \\
\hline \multicolumn{4}{|c|}{ Nodal status } \\
\hline Negative & $397(48.2 \%)$ & $427(51.8 \%)$ & \multirow{2}{*}{0.052} \\
\hline Positive & $222(42.6 \%)$ & $299(57.4 \%)$ & \\
\hline \multicolumn{4}{|c|}{ Nottingham Prognostic Index } \\
\hline Good prognostic group & $266(61.4 \%)$ & $167(38.6 \%)$ & \multirow{3}{*}{$<0.0001$} \\
\hline Moderate prognostic group & $283(41.5 \%)$ & $399(58.5 \%)$ & \\
\hline Poor prognostic group & $70(46.1 \%)$ & $159(69.4 \%)$ & \\
\hline \multicolumn{4}{|c|}{ Histological grade } \\
\hline Grades 1 and 2 & $427(59.2 \%)$ & $294(40.8 \%)$ & \multirow{2}{*}{$<0.0001$} \\
\hline Grade 3 & $194(30.9 \%)$ & $433(69.1 \%)$ & \\
\hline \multicolumn{4}{|c|}{ Lymphovascular invasion } \\
\hline Negative & $455(47.7 \%)$ & $498(52.3 \%)$ & \multirow{2}{*}{0.063} \\
\hline Positive & $166(42.0 \%)$ & $229(58.0 \%)$ & \\
\hline \multicolumn{4}{|c|}{ Oestrogen receptor } \\
\hline Negative & $69(24.4 \%)$ & $214(75.6 \%)$ & \multirow{2}{*}{$<0.0001$} \\
\hline Positive & $553(51.8 \%)$ & $514(48.2 \%)$ & \\
\hline
\end{tabular}


Supplementary Table 2 Survival analysis based on clinicopathological characteristics of breast cancer, including LCN2 nuclear expression

\begin{tabular}{|c|c|c|c|c|}
\hline \multirow{2}{*}{\multicolumn{2}{|c|}{ Factors }} & \multicolumn{3}{|c|}{ Multivariate analysis } \\
\hline & & Hazard Ratio & $95 \% \mathrm{CI}$ & $p$ value \\
\hline \multirow{2}{*}{ LCN2 nuclear expression } & Low & \multicolumn{3}{|c|}{ Reference } \\
\hline & High & 0.88 & $0.68-1.15$ & 0.36 \\
\hline \multirow{2}{*}{ Tumour size } & $<2 \mathrm{~cm}$ & \multicolumn{3}{|c|}{ Reference } \\
\hline & $\geq 2 \mathrm{~cm}$ & 1.38 & $1.05-1.79$ & 0.018 \\
\hline \multirow{2}{*}{ Nodal status } & Negative & \multicolumn{3}{|c|}{ Reference } \\
\hline & Positive & 2.11 & $1.79-2.48$ & $<0.0001$ \\
\hline \multirow{2}{*}{ Histological grade } & Low & \multicolumn{3}{|c|}{ Reference } \\
\hline & High & 1.91 & $1.51-2.41$ & $<0.0001$ \\
\hline
\end{tabular}




\begin{tabular}{|c|c|c|c|}
\hline \multicolumn{4}{|c|}{ Progesterone receptor } \\
\hline Negative & $201(35.7 \%)$ & $362(64.3 \%)$ & \multirow{2}{*}{$<0.0001$} \\
\hline Positive & $417(53.6 \%)$ & $361(46.4 \%)$ & \\
\hline \multicolumn{4}{|c|}{ Human epidermal growth factor receptor 2} \\
\hline Negative & $555(47.6 \%)$ & $610(52.4 \%)$ & \multirow{2}{*}{0.0042} \\
\hline Positive & $67(36.2 \%)$ & $118(63.8 \%)$ & \\
\hline \multicolumn{4}{|c|}{ Breast cancer subtypes } \\
\hline Triple negative & $53(25.5 \%)$ & $155(74.5 \%)$ & \multirow{2}{*}{$<0.0001$} \\
\hline Non-triple negative & $569(49.8 \%)$ & $573(50.2 \%)$ & \\
\hline
\end{tabular}


a)

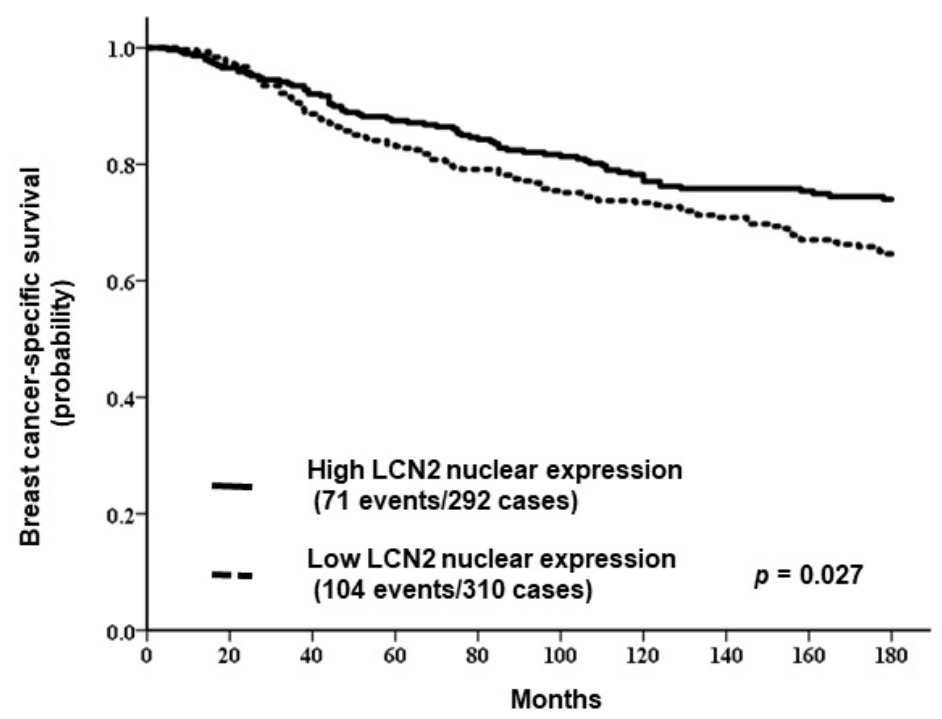

b)

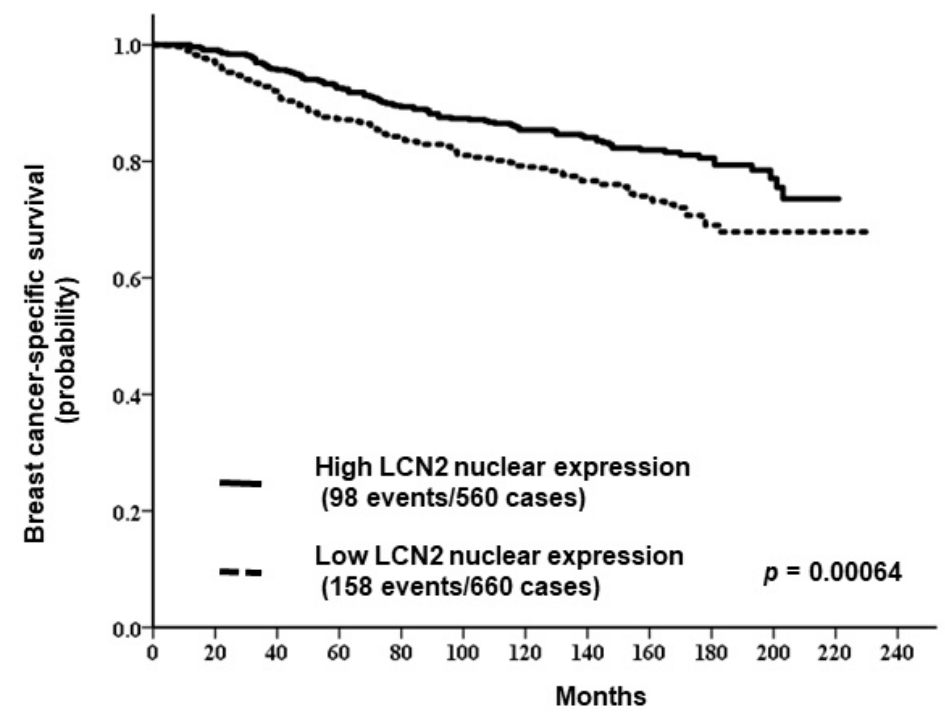

Fig 3 
a)

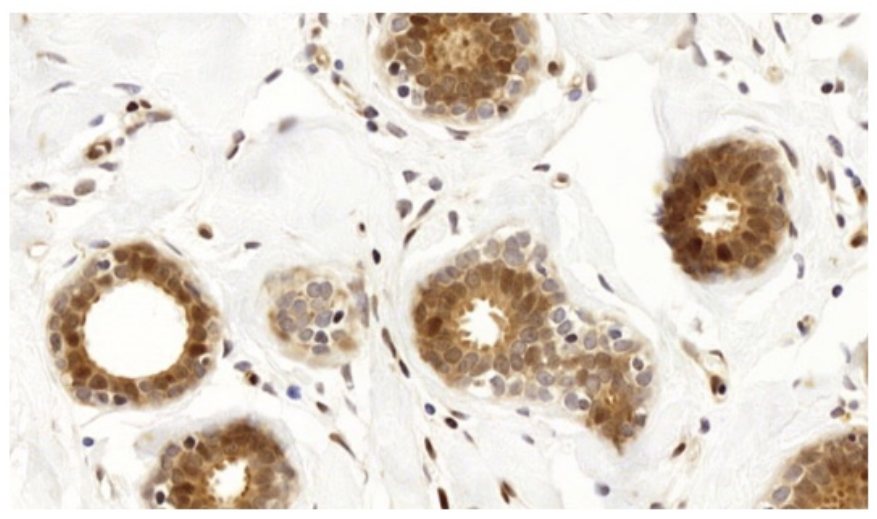

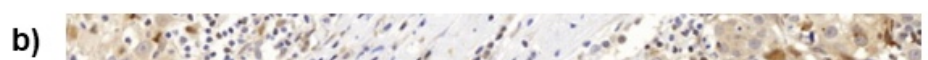

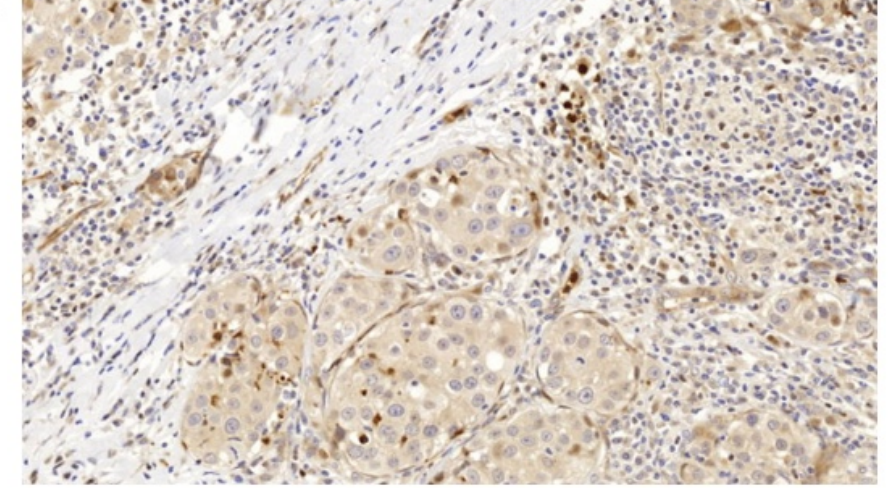

c)

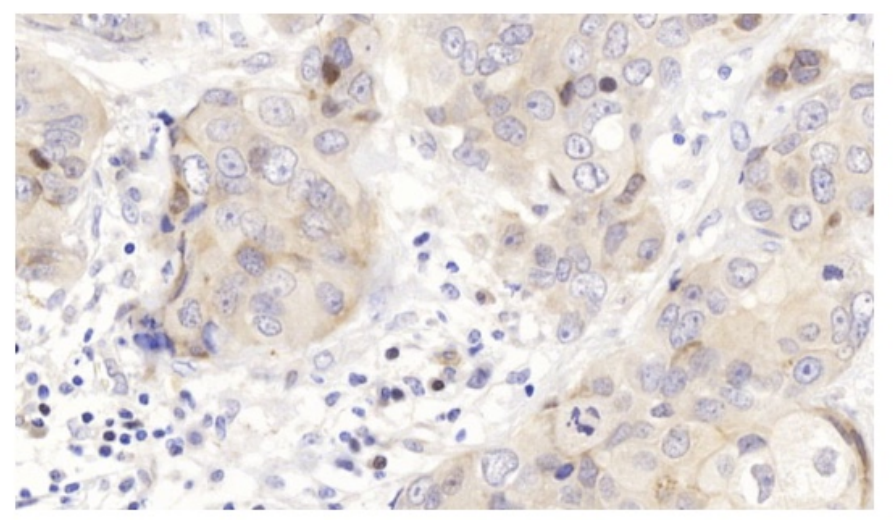

Fig 1 
a)

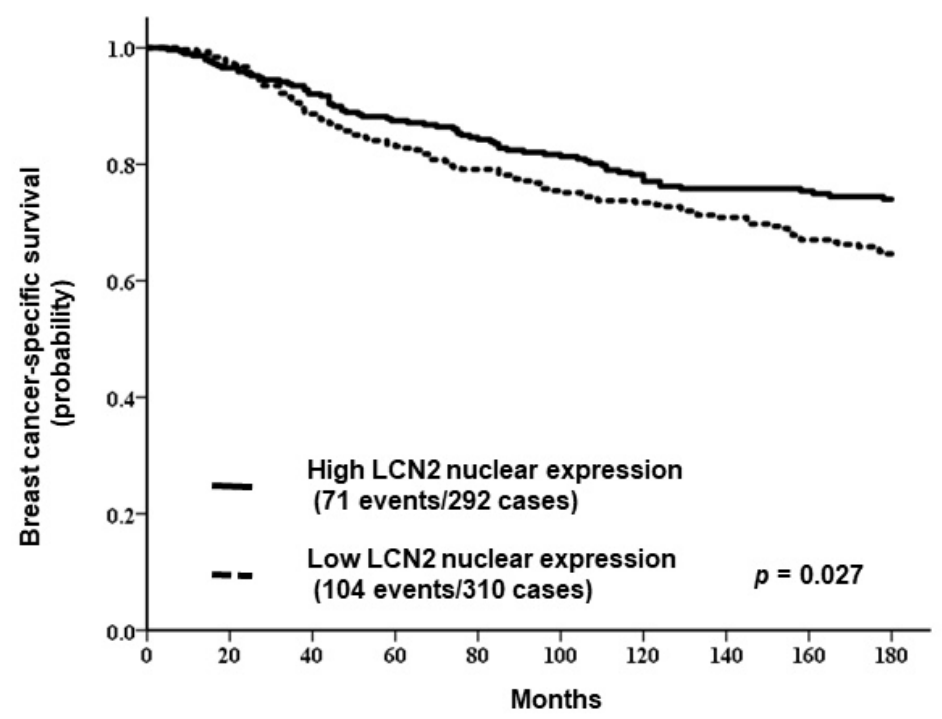

b)

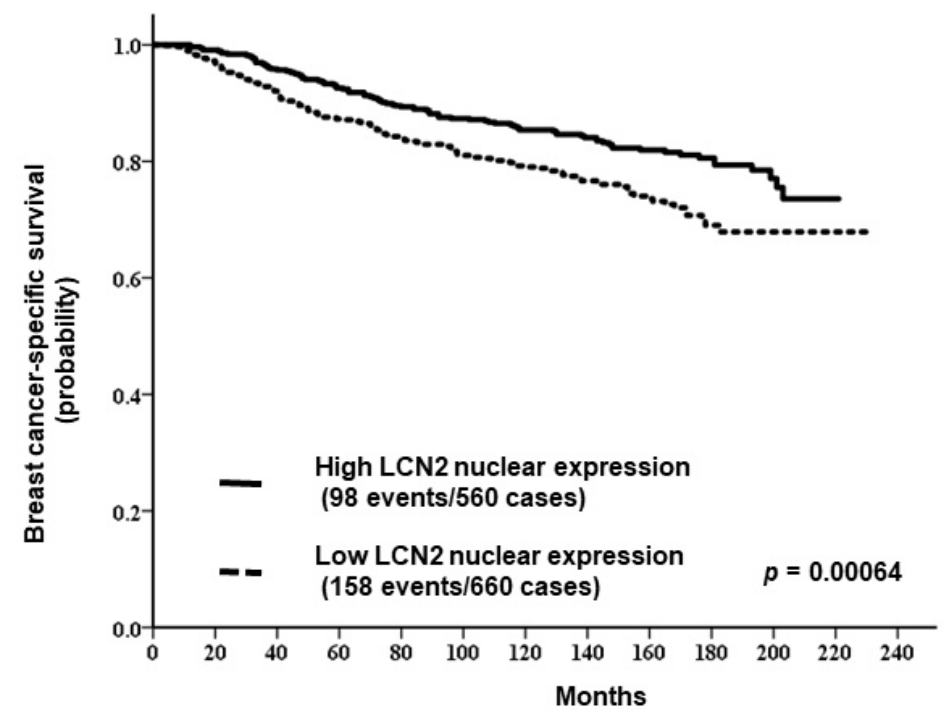

Fig 3 
a)

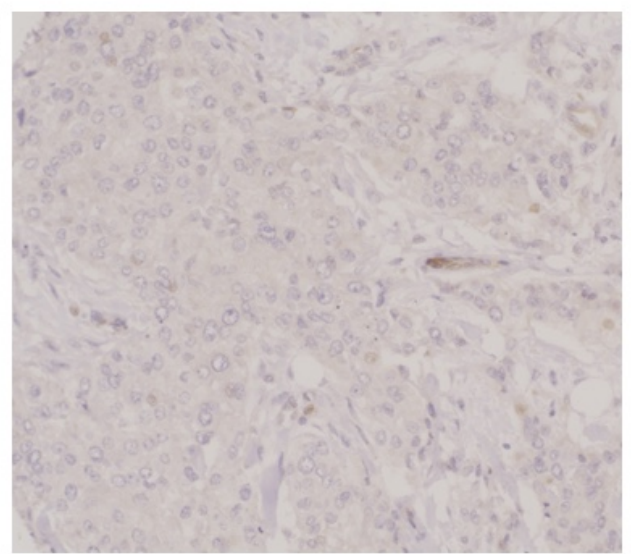

b)

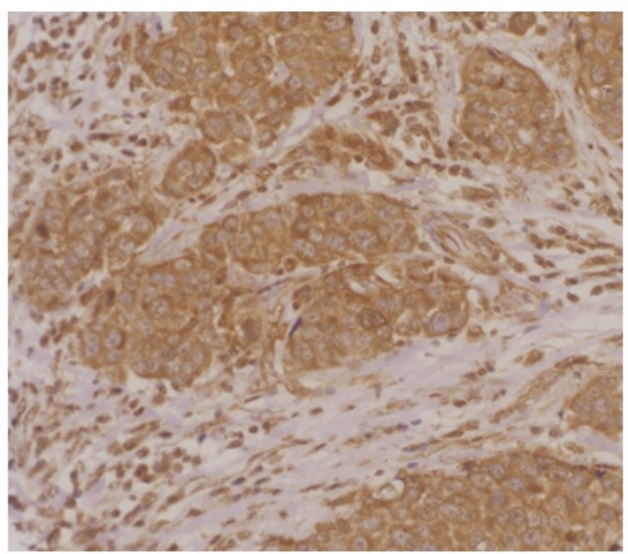

c)

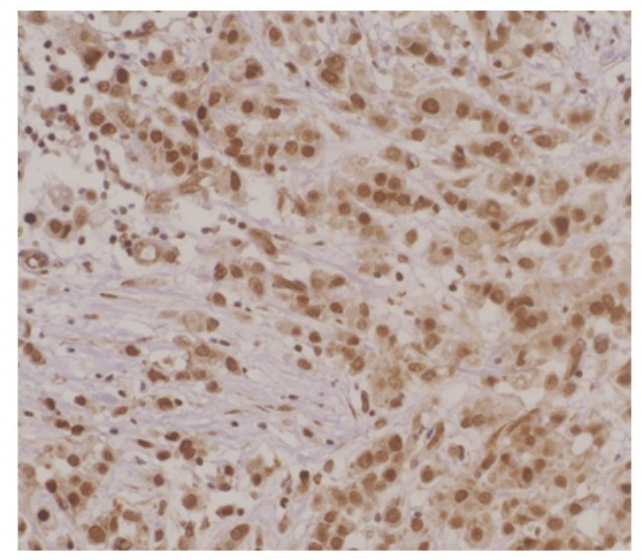

d)

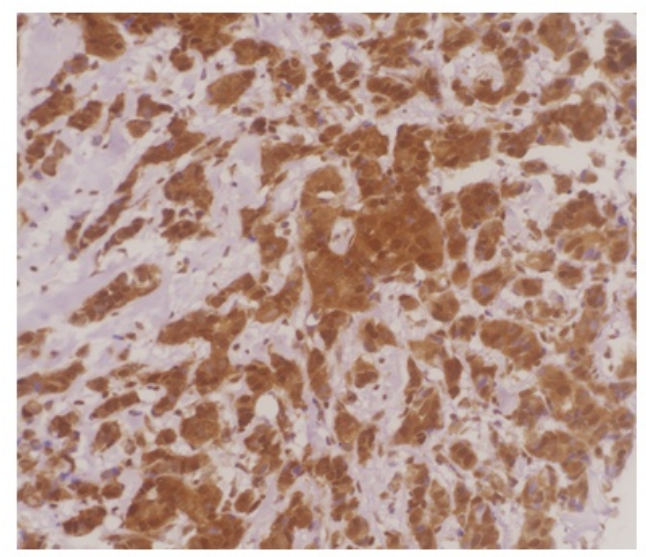

Fig 2 


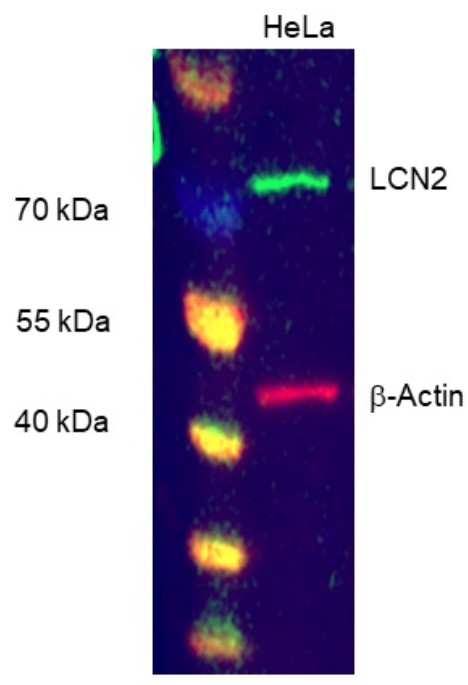

Supplementary Fig 1 


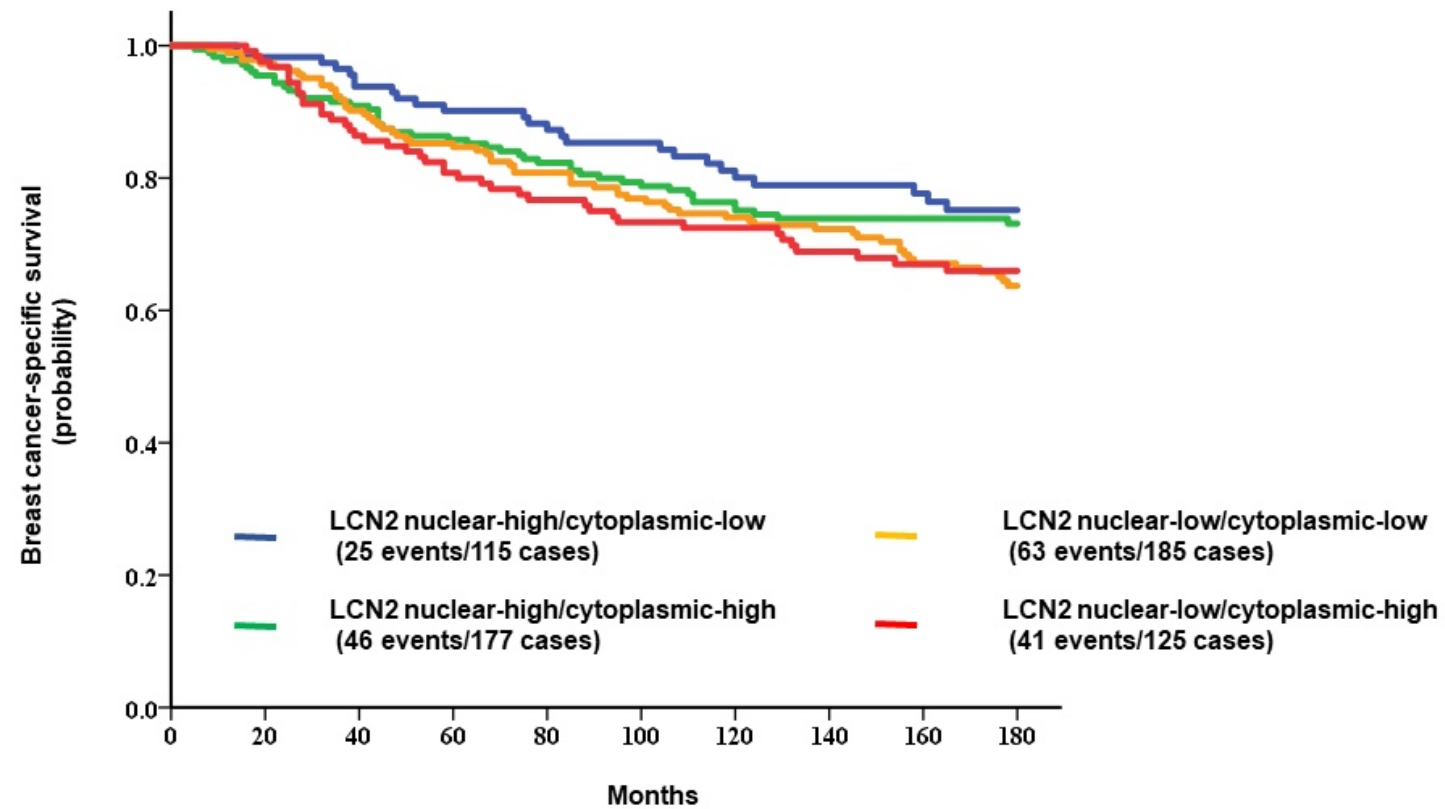

LCN2 nuclear-high/cytoplasmic-low vs. LCN2 nuclear-high/cytoplasmic-high: $p=0.48$ LCN2 nuclear-high/cytoplasmic-low vs. LCN2 nuclear-low/cytoplasmic-low: $p=0.056$ LCN2 nuclear-high/cytoplasmic-low vs. LCN2 nuclear-low/cytoplasmic-high: $p=0.079$

\section{Supplementary Fig 3}




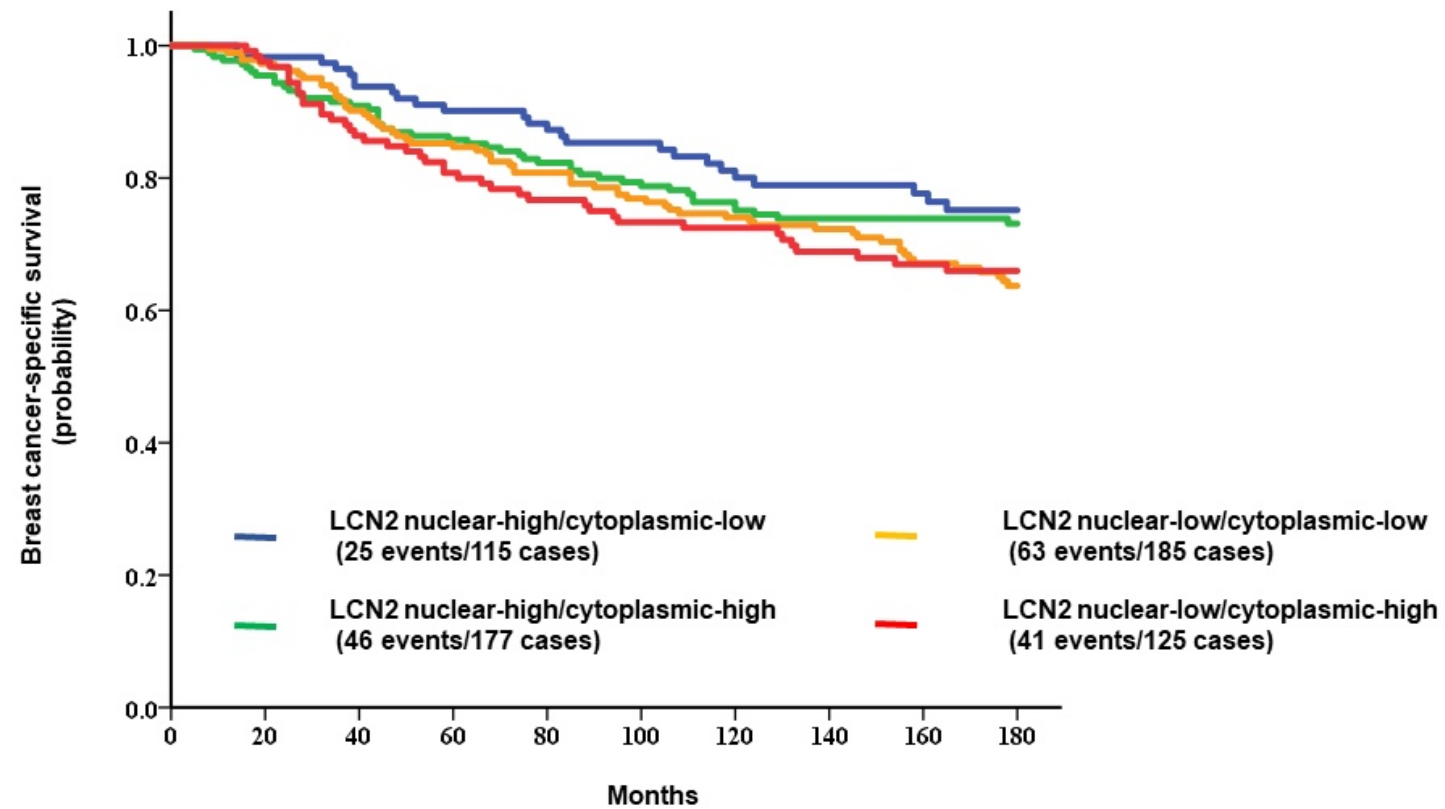

LCN2 nuclear-high/cytoplasmic-low vs. LCN2 nuclear-high/cytoplasmic-high: $p=0.48$ LCN2 nuclear-high/cytoplasmic-low vs. LCN2 nuclear-low/cytoplasmic-low: $p=0.056$ LCN2 nuclear-high/cytoplasmic-low vs. LCN2 nuclear-low/cytoplasmic-high: $p=0.079$

\section{Supplementary Fig 3}




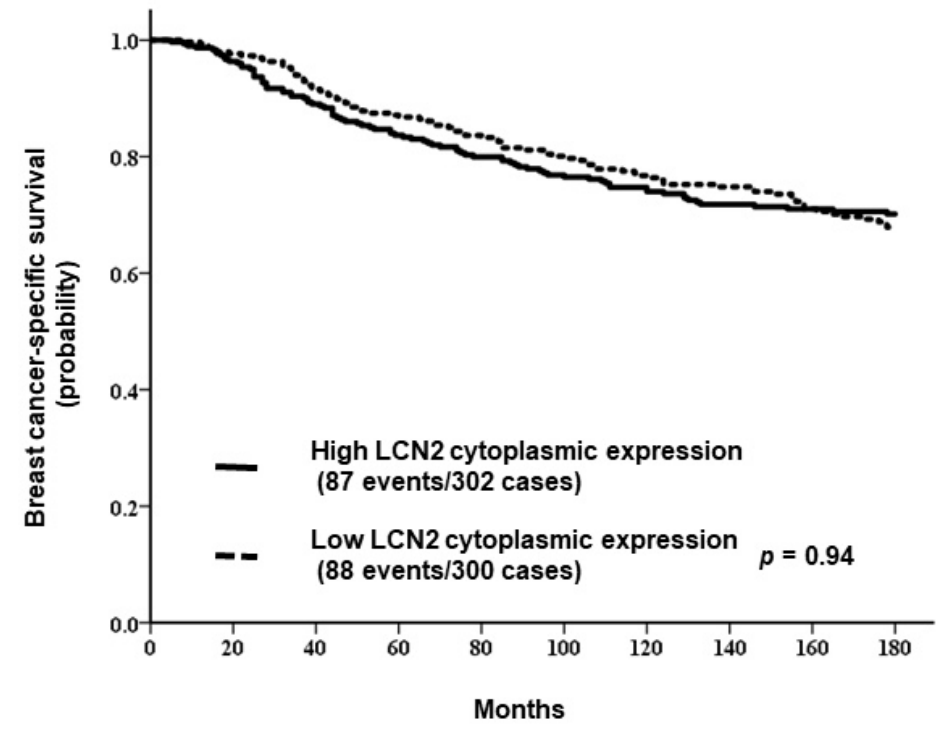

Supplementary Fig 2 